The first experiment of a THz gyrotron with a pul se magnet

\begin{tabular}{|l|l|}
\hline 著者 & $\begin{array}{l}\text { I dehar a T, Tsuchi ya H, Wat anabe O, Agusu La, } \\
\text { M t sudo S }\end{array}$ \\
\hline $\begin{array}{l}\text { j our nal or } \\
\text { publ i cat i on ti t l e }\end{array}$ & $\begin{array}{l}\text { I nt er nat i onal J our nal of I nf r ar ed and } \\
\text { M I I i net er Waves }\end{array}$ \\
\hline vol une & 27 \\
\hline number & 3 \\
\hline page r ange & $319-331$ \\
\hline year & $2006-03$ \\
\hline URL & ht t p: //hdl . handl e. net /10098/1862 \\
\hline
\end{tabular}




\title{
THE FIRST EXPERIMENT OF
}

\section{A THz GYROTRON WITH A PULSE MAGNET}

\author{
T. Idehara, H. Tsuchiya, O. Watanabe, La Agusu and *S. Mitsudo \\ Research Center for Development of Far Infrared Region, University of Fukui \\ (FIR FU) \\ Bunkyo 3-9-1, Fukui-shi 910-8507, Japan
}

Received 18 January, 2006

\begin{abstract}
A $\mathrm{THz}$ gyrotron with a pulse magnet has been designed, constructed and operated in FIR FU. It is developed as one of high frequency gyrotrons included in Gyrotron FU Series. The gyrotron has already achieved the first experimental result for high frequency operations whose radiation frequency exceeds $1 \mathrm{THz}$. In this paper, the design detail and the operation test results for sub-terahertz to terahertz range are described. The second harmonic operation is confirmed experimentally at the expected frequency of $1.005 \mathrm{THz}$ due to $\mathrm{TE}_{6,11}$ cavity mode at the magnetic field intensity of $19.0 \mathrm{~T}$.
\end{abstract}

Key words: Gyrotron, Pulse magnet, Terahertz radiation, Gyrotron FU Series, Second harmonic operation

\section{Introduction}

Gyrotron development is being advanced in two ways. The major way is development of high power, millimeter wave gyrotrons for heating and current drive for fusion plasma. It is going world-widely and has achieved around $1 \mathrm{MW}$ output power for long pulse operation (longer than several tens second or quasi $\mathrm{CW}$ ) at the frequency of $170 \mathrm{GHz}$ or $140 \mathrm{GHz}^{1)}$. On the other hand, medium power, high frequency gyrotrons are being developed in several institutions in the world ${ }^{2)-5)}$. In the cases, high magnetic field and higher harmonic operations are used for increasing operation frequency. Such

*Present address: Cryogenic Laboratory, Faculty of Engineering, University of Fukui, Bunkyo 3-9-1, Fukui-shi 910-8507, Japan 
gyrotrons have already covered wide frequency range in millimeter to submillimeter wavelength region ${ }^{6,7)}$ and been applied as submillimeter wave radiation sources for wide fields including plasma diagnostics ${ }^{8)}$, 9), electron spin resonance (ESR) specroscopy ${ }^{10)}$, new medical technology ${ }^{11}$, and so on. These gyrotrons are only one high power stable $\mathrm{THz}$ radiation source and will be important and useful in future for development of high power $\mathrm{THz}$ technologies.

Our gyrotrons developed in FIR FU named Gyrotron FU Series belongs to the second type gyrotrons, that is, medium power high frequency gyrotrons. The series has achieved following items, 1) frequency step-tuneability in wide range in millimeter to submillimeter wavelength region (from 38 to 889 $\mathrm{GHz})^{12)}$,2) highest frequency $(889 \mathrm{GHz})$ corresponding to the wavelength of $377 \mu \mathrm{m}$ by using second harmonic operation at the field intensity of around $17 \mathrm{~T}, 3$ ) modulation of amplitude and frequency of the output ${ }^{13), 14}$, 4) stabilization of amplitude and frequency ${ }^{15)}$, 5) higher harmonic operations up to fifth ${ }^{16)}$ and 6) high-purity mode operations at many cavity modes ${ }^{17)}$ by installation of a carefully designed cavity. Also, we have achieved mode conversion from circular waveguide modes to a Gaussian mode for applications of our gyrotrons to many fields ${ }^{18)}$.

However, up to the present, gyrotrons have not achieved the operation at the frequency of one terahertz. This paper presents the results of the first experiment of a $\mathrm{THz}$ gyrotron which realizes the breakthrough. In the next section, an experimental setup and procedures are presented in brief, in section 3, the simulation results of a $\mathrm{THz}$ gyrotron is described, in section 4 , experimental results and considerations comparing with the simulation results are presented in detail and in section 5 , the contents of the paper are summarized.

\section{Experimental setup and procedures}

A $\mathrm{THz}$ gyrotron consists of a gyrotron tube with high $\mathrm{Q}$ value for high frequency cavity modes, an ice-protecting pulse magnet whose maximum field intensity is around $21.4 \mathrm{~T}, 300 \mathrm{~kJ}$ capacitor bank for operating the pulse magnet, power supplies for a triode magnetron injection gun and gun coils and a controlling system for the whole gyrotron system. Fig. 1 shows the block diagram of the whole system of the THz gyrotron.

An ice-protecting pulse magnet is installed on the second floor. The cross 


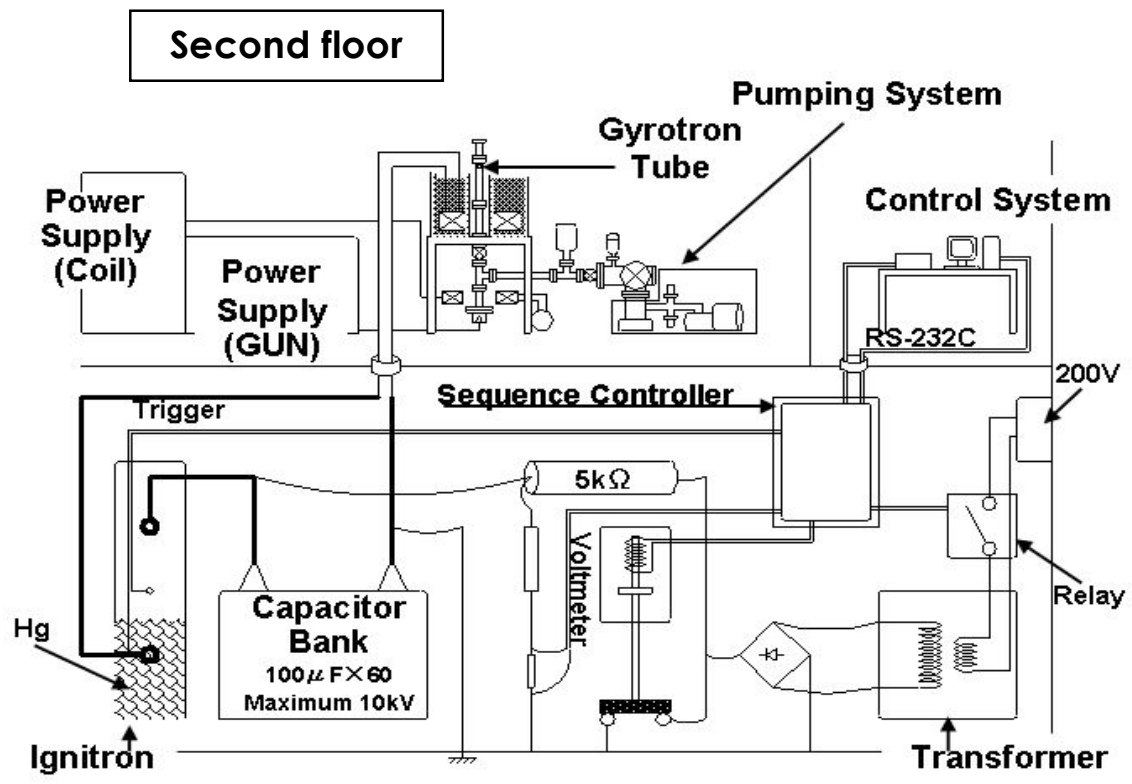

First floor

Fig. 1 The block diagram of the whole system of a THz gyrotron.

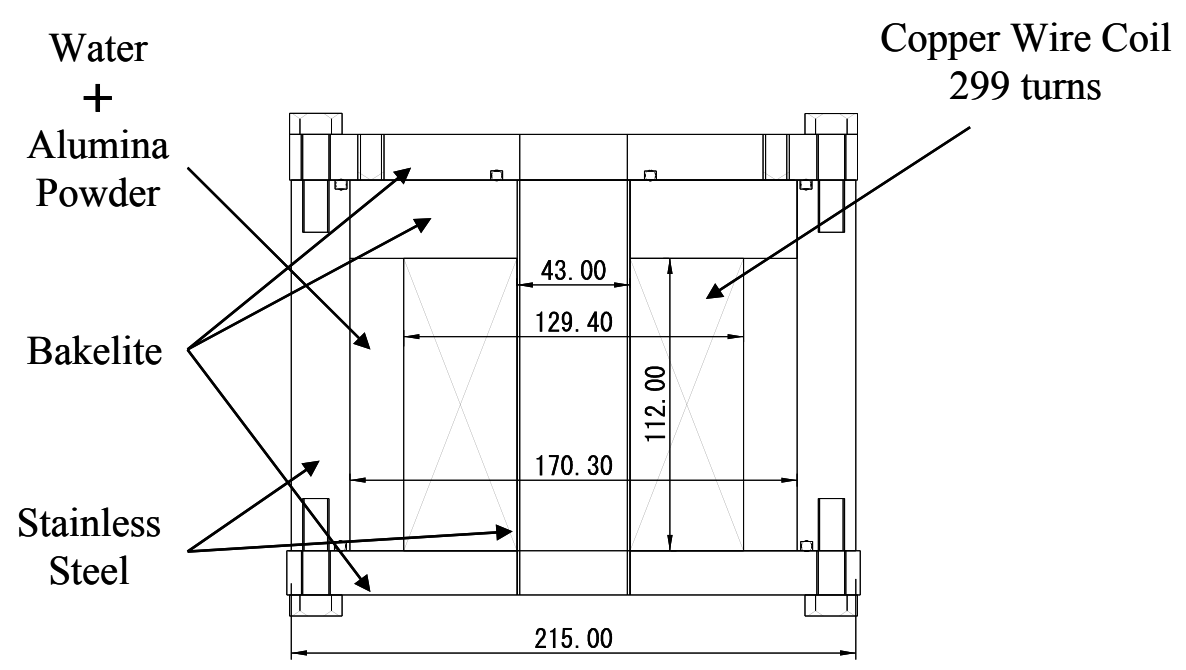

Fig. 2 The cross section of an ice-protecting pulse magnet installed on the second floor.. 
section is shown in Fig. 2. It is a simple solenoid coil. Copper wire is wound in 299 turns. The inner diameter, outer diameter and length are $43 \mathrm{~mm}, 129.4$ $\mathrm{mm}$ and $112 \mathrm{~mm}$, respectively. The coil is inserted in stainless steel cylinder whose inner diameter and thickness are $170.3 \mathrm{~mm}$ and $22.4 \mathrm{~mm}$, respectively. Water with alumina powder is surrounding the coil and fulfill the clearance between the coil and a stainless steel cylinder. The whole system of an ice-protecting pulse magnet is installed in a cryostat and cooled down by liquid nitrogen. Then, water with alumina powder is frozen and fixes the solenoid coil tightly in order to protect the coil from expanding in radial direction during pulse operation.

The pulse magnet is operated by use of a capacitor bank installed on the first floor. The total capacitance is $6 \mathrm{mF}$ and maximum biased voltage is $10 \mathrm{kV}$. Therefore, the maximum stored energy is $300 \mathrm{~kJ}$. It is discharged through an ignitron and applied to the pulse magnet. The field intensity is increased up to $21 \mathrm{~T}$ at the biased voltage of $5.7 \mathrm{kV}$ and the pulse half-value width is typically, around $11 \mathrm{~ms}$. The gyrotron operates in short pulse which is limited by a high voltage power supply. A typical pulse width of the output of the supply is 1 $\mathrm{ms}$. The timing of the pulsed operation is controlled by the controlling system from the second floor. In addition to the pulse magnet, three pieces of water-cooled copper coils are installed in the gun region for controlling parameters of electron beam.

A demountable gyrotron tube is inserted on the axis of the magnet. All of the components of the gyrotron, for example, an electron gun, a resonant cavity, an output window and a transmission waveguide from the cavity to the output window. Fig. 3 shows the cross section of the gyrotron tube with a pulse magnet system. A vacuum layer of the cryostat is expanded to inside of the gyrotron tube. Usually, the pressure in the tube is less than $10^{-7}$ Torr.

We are using a triode magnetron injection gun in order to control the beam parameter for modulation of amplitude and frequency. Mirror ratio defined as $B_{c} / B_{g}$ is varied from 84 to 127 , where $B_{c}$ and $B_{g}$ are magnetic field intensities at cavity region and gun region, respectively.

A cavity is a simple cylindrical one whose inner diameter and length are 3.9 $\mathrm{mm}$ and $10 \mathrm{~mm}$, respectively. One candidate of cavity mode which will achieve the breakthrough of $1 \mathrm{THz}$ at the second harmonic is $\mathrm{TE}_{4,12}$. Corresponding frequency is $1.03 \mathrm{THz}$. The field intensity for the operation is 19.2 T. Such a high field could be realized by the ice-protecting pulse magnet. 


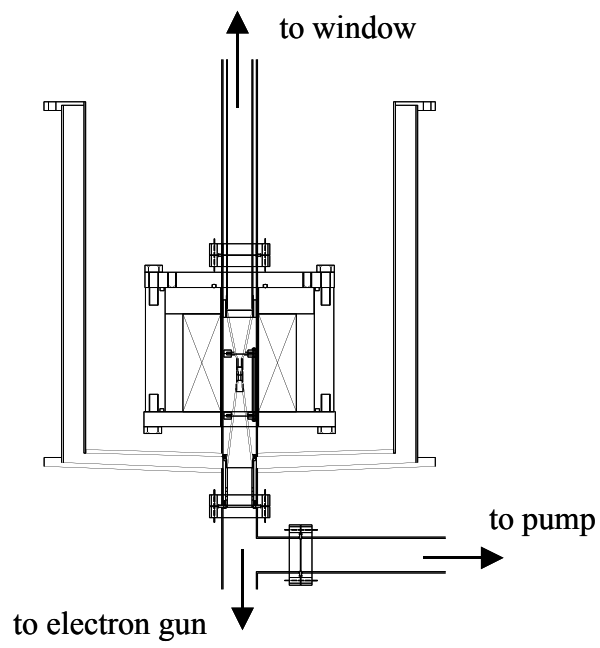

Fig. 3 The cross section of the gyrotron tube with an ice-protecting pulse magnet system

The output window consists of alumina disk with the thickness of $3 \mathrm{~mm}$. An output power from the cavity penetrates through the circular waveguide (the diameter is $28 \mathrm{~mm}$ ) and the window, and then comes out to the external waveguide whose diameter is $28 \mathrm{~mm}$.

\section{Simulation results}

First of all, we tried to estimate the field intensity profile in cavity region which is generated by the pulse magnet. The effect of eddy current induced on the cavity wall makes the profile asymmetric. However, the uniformity in the cavity region is kept at around 1 percent.

Next, simulations for trajectories of beam electrons are carried out for a real triode magnetron injection gun and field configuration. The results show that almost all electrons penetrate through the cavity region toward the collector. Obtained typical beam electron parameters at entrance of the cavity are as follows, the pitch factor is around 1.2, the spread of perpendicular velocity 5 percent, the energy of the electron $30 \mathrm{keV}$ and beam current 150 $\mathrm{mA}$.

Finally, we tried the estimation of starting current of each cavity mode operation at both fundamental and the second harmonic resonances. The results are shown in Fig. 4. There are many possible cavity modes excited in 
the beam current range below $100 \mathrm{~mA}$ in both fundamental and the second harmonic cases.



Fig. 4 Starting current $I$ of each cavity mode operation at the fundamental or the second harmonic resonance as a function of magnetic field $B$.

\section{Experimental results band considerations}

Our first objective of the experiment is achievement of the breakthrough of $1 \mathrm{THz}$, the renewal of the highest frequency of gyrotron by using high magnetic field of a pulse magnet and the second harmonic operation. Therefore, it is most important how high field our pulse magnet will arrive at.

Fig. 5 shows results of operation test of the pulse magnet. In the measurement, the coil current is observed by a pick-up coil installed inside of a cavity and integrated to give the variation of field intensity. The parameter is a biased voltage of the capacitor bank. The maximum field intensity is increased up to $19.1 \mathrm{~T}$, which is comparable with the simulation results. The half value width of the pulse field is around $11 \mathrm{~ms}$. Such a measurement was carried out before pumping out the gyrotron tube.

Next, we tried the operation test of the gyrotron by use of a high voltage pulse supply whose typical pulse length is $1 \mathrm{~ms}$. As demonstrated by the 


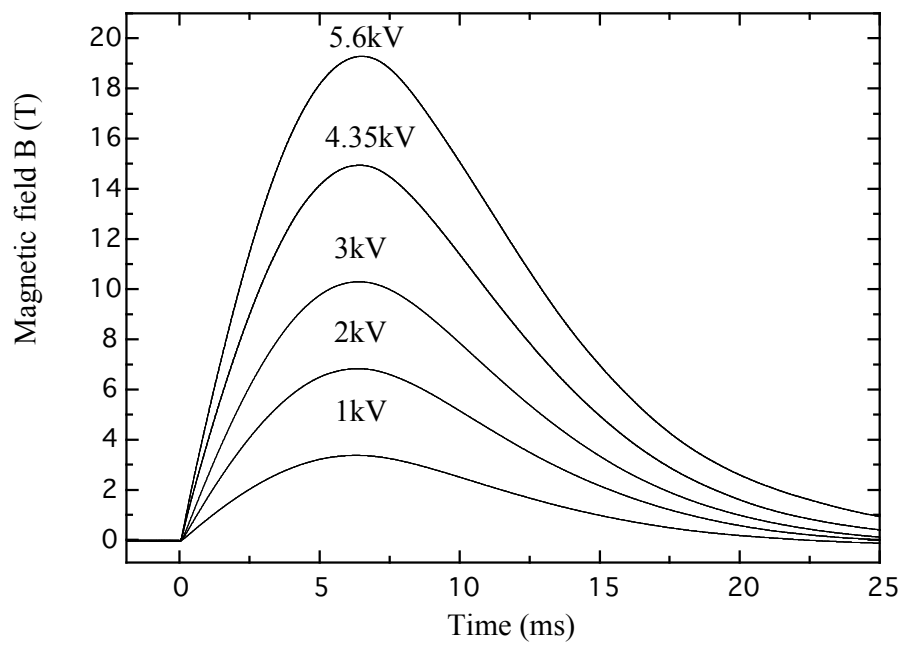

Fig. 5 The measurement results of variation of field intensity $B$ with a biased voltage of the capacitor bank as a parameter.

estimation of starting current (Fig. 4), there are many cavity mode expected to be excited in both fundamental and second harmonic in the region of magnetic field intensity which is generated by an ice-protecting pulse magnet. We should use high pass filter at the end of external waveguide system for separation of the rather week second harmonic operation mode from stronger fundamental mode. The filter is only a narrow hole which is recognized as a thin circular waveguide having a cutoff frequency corresponding to the fundamental $\mathrm{TE}_{11}$ waveguide mode. Typically, we used narrow holes with diameters of $0.5 \mathrm{~mm}$ and $0.3 \mathrm{~mm}$. Corresponding cutoff frequencies are 352 $\mathrm{GHz}$ and $586 \mathrm{GHz}$, respectively.

One of measurement results for operation of a $\mathrm{THz}$ gyrotron is demonstrated in Fig. 6. Radiation power detected by InSb cooled by liquid helium is traced as functions of magnetic field in both upper and lower traces of Fig. 6. Radiation power in the lower trace is detected after a high pass filter with a hole of $0.5 \mathrm{~mm}$ in diameter, while radiation power in the upper trace after a high pass filter with hole of $0.3 \mathrm{~mm}$ in diameter. The magnetic field intensity is varied from $14.5 \mathrm{~T}$ to $15.9 \mathrm{~T}$ in the time interval $(1 \mathrm{~ms})$ of the high voltage pulse applied to a gun cathode. During the interval, corresponding frequencies of fundamental and second harmonic operations vary from 383 
$\mathrm{GHz}$ to $421 \mathrm{GHz}$ and from $767 \mathrm{GHz}$ to $841 \mathrm{GHz}$, respectively. Therefore, radiation power detected after $0.3 \mathrm{~mm}$ filter includes only second harmonic

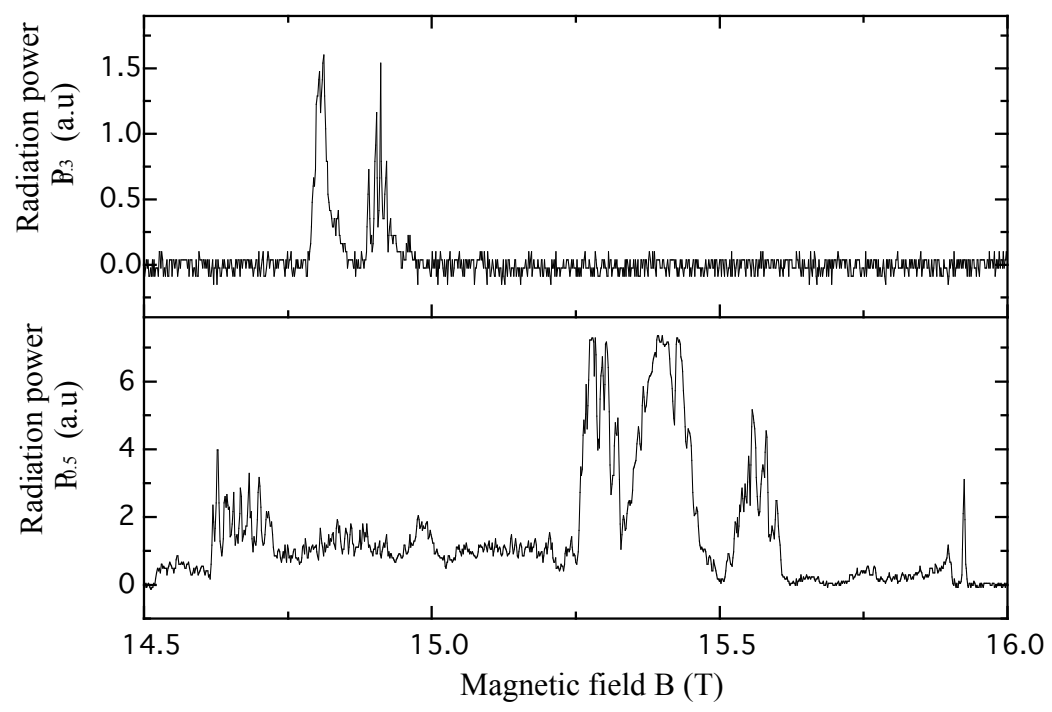

Fig. 6 Radiation power measured by use of an InSb detector cooled by liquid helium as magnetic field generated by a pulse magnet. Upper trace: Radiation power $P_{0.3}$ after a high pass filter with a hole of $0.3 \mathrm{~mm}$ in diameter which includes only second harmonic operations. Lower trace: Radiation power $P_{0.5}$ detected after a high pass filter with a hole of $0.5 \mathrm{~mm}$ in diameter which includes both the fundamental and the second harmonic operations.

radiations, while radiation power detected after $0.5 \mathrm{~mm}$ filter both fundamental and second harmonic radiations. In the upper trace of Fig. 6, the magnetic field corresponding to the observed radiation peaks are $14.8 \mathrm{~T}$ and 14.9 T. Therefore, this means that the second harmonic operations occur at the frequencies of $783 \mathrm{GHz}$ and $789 \mathrm{GHz}$, which are almost same as the frequency of $\mathrm{TE}_{0,10}$ or $\mathrm{TE}_{2,10}$ cavity mode. It is expected from the calculation results for starting current, that these mode has a possibility of mode competition with $\mathrm{TE}_{4,4}$ or $\mathrm{TE}_{2,5}$ fundamental cavity modes.

Fig. 7 shows the result of a similar measurement where the field intensity is increased further. The radiation pulse observed in the middle trace results 
from second harmonic operation, because it penetrates through a high-pass filter with a hole of $0.3 \mathrm{~mm}$ in diameter. The magnetic field corresponding to

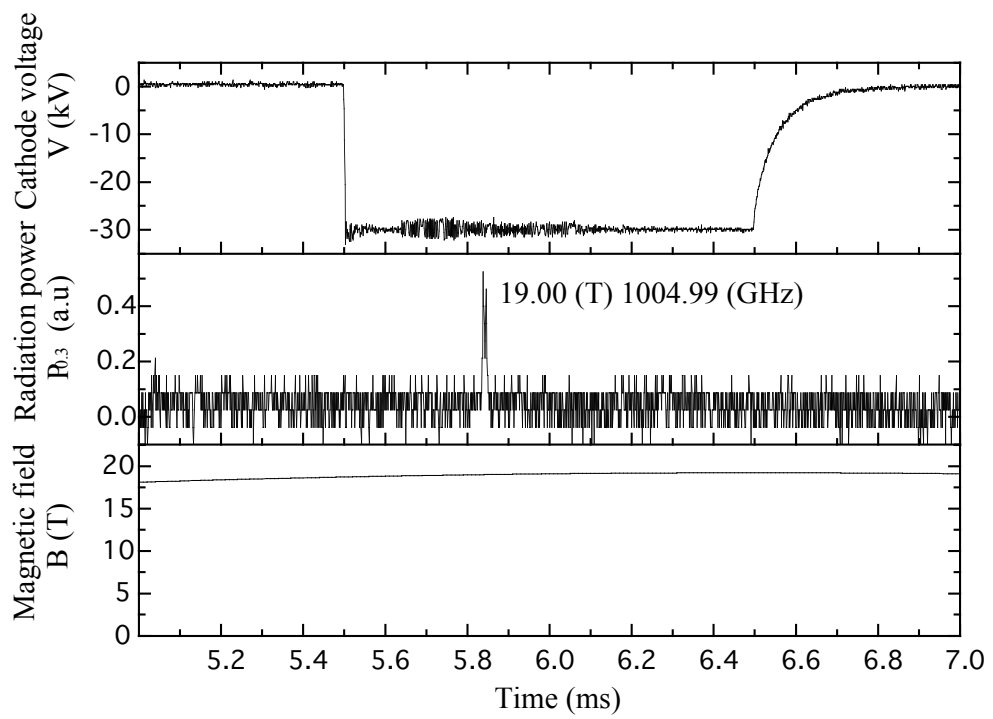

Fig. 7 Radiation power $P_{0.3}$ measured as a function of time by use of an $\mathrm{InSb}$ detector cooled by liquid helium with variation of magnetic field generated by a pulse magnet $B$ and cathode voltage $V$. The radiation pulse observed in the upper trace results from second harmonic operation, which penetrates through a high pass filter with a hole of $0.3 \mathrm{~mm}$ in diameter.

the observed radiation peak is $19.0 \mathrm{~T}$. Therefore, the second harmonic operation occurs at the frequency of $1.005 \mathrm{THz}$ which is almost same as the frequency of $\mathrm{TE}_{6,11}$ cavity mode. It is expected from Fig. 5, that this mode has a possibility of mode competition with a fundamental mode $\mathrm{TE}_{55}$.

Similar measurements have been carried out in wide range of magnetic field intensity. In any cases, second harmonic operations were separated from the fundamental operations by using high pass filters. Many operation modes at the fundamental and the second harmonics were already found. The expected frequencies of observed radiation peaks estimated from corresponding field intensities are plotted in Fig. 8 as functions of magnetic fields. Frequency step tuneability is achieved in the frequency ranges from $387 \mathrm{GHz}$ to $471 \mathrm{GHz}$ by fundamental operations and from $711 \mathrm{GHz}$ to 
$1009 \mathrm{GHz}$ by second harmonic operations. The highest frequency has achieved the breakthrough of $1 \mathrm{THz}$. This is the first experiment for a $\mathrm{THz}$ gyrotron using high magnetic field exceeding $19.1 \mathrm{~T}$ and second harmonic operation.

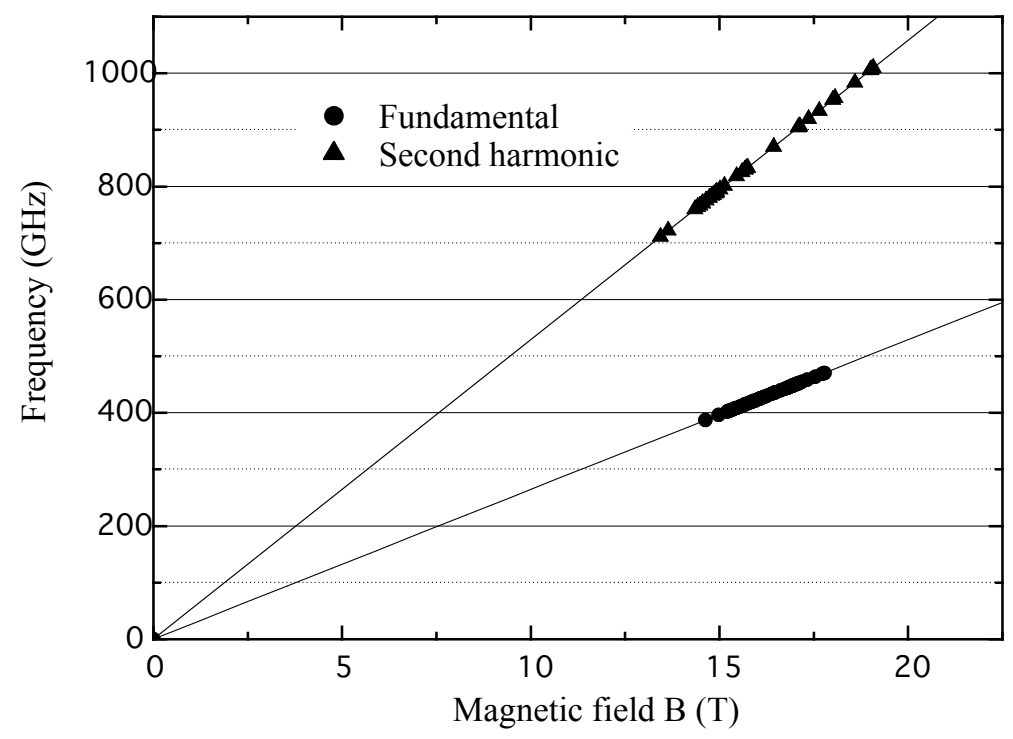

Fig. 8 Expected frequencies of observed radiation peaks at the fundamentals and the second harmonics as functions of magnetic fields $B$.

\section{Summary}

Design, construction and operation test of a $\mathrm{THz}$ gyrotron with high field pulse magnet has been carried out successfully. The gyrotron operates at both fundamental and second harmonics of electron cyclotron frequency. It has achieved frequency step tuneability in wide frequency range from $387 \mathrm{GHz}$ to $1009 \mathrm{GHz}$ and the highest frequency exceeds $1 \mathrm{THz}$. This is the first experiment of a THz gyrotron which achieved the breakthrough of $1 \mathrm{THz}$. The gyrotron renews the record of high frequency operation of gyrotron. The experimental results presented in this paper are just only preliminary ones. We will publish detailed experimental results soon after clarifying dependencies of radiation power on various experimental parameters and comparing with simulation results. 


\section{Acknowledgements}

This work was supported by the special fund from the President of University of Fukui, Professor Shinpei Kojima, named Project Allocation Fund of University of Fukui. The authors would thank to Mr. T. Kanemaki for his technical supports concerning construction of the gyrotron itself and measurement equipments.

\section{References}

1) For example, G.Dammertz, et al., Experimental results on the $140 \mathrm{GHz}, 1$ MW, CW gyrotrons for the stellarator W7-X, Digest of the Joint 30th Int. Conf. on Infrared and Millimeter Waves and 13th Int. Conf. on Terahertz Electronics, Williamsburg, USA, September 19-23 2005, pp. 235-236.

2) G.F. Brand, Z. Chen, N.G. Douglas, M. Cross, J.Y.L. Ma and L.C. Robinson, A tunable millimeter-submillimeter gyrotron, Int. J. Electronics $\mathbf{5 7}$ (1984) 863-870.

3) S.E. Spira-Hakkarainen, K.E. Kreischer and R. J. Temkin, Submillimeter-wave harmonic gyrotron experiment, IEEE Trans. Plasma Sci., PS-18 (1990) 334-342.

4) T. Idehara, T. Tatsukawa, I. Ogawa, H. Tanabe, T. Mori, S. Wada, G.F. Brand and M.H. Brennan, Development of a Second Cyclotron Harmonic Gyrotron Operating at Submillimeter Wavelengths, Phys. of Fluids B4 (1992) 267-273.

5) V.A. Flyagin, A.G. Luchinin, G.S. Nusinovich, Submillimeter-wave gyrotrons: theory and experiment, Int. J. Infrared and Millimeter Waves 4 (1983) 629-637.

6) T. Idehara, T. Tatsukawa, I. Ogawa, Y. Shimizu, and T. Kanemaki , Development of a High-Frequency, Second-Harmonic Gyrotron Tunable up to $636 \mathrm{GHz}$, Phys. of Fluids $\mathbf{B 5}$ (1993) 1377-1379.

7) K.D. Hong, G.F. Brand and T. Idehara, A 150-600 GHz Step-Tunable Gyrotron, J. Appl. Phys. 74 (1993) 5250-5258.

8) Y. Terumichi, S. Kubo, A. Ando, Y. Yanagimoto, K. Ogura, H. Tanaka, J. Takahashi, I. Tonai, M. Nakamura, T. Maekawa, S. Tanaka and T. Idehara, Study of low frequency density fluctuations in the WT-2 tokamak by mm and 
submm wave scattering, Digest of Ninth Int. Conf. on Infrared and Millimeter Waves, Takarazuka, Japan, October 22-29, 1984, pp. 411-412.

9) T. Idehara, I. Ogawa, K. Kawahata, H. Iguchi and K. Matsuoka, Application of the Gyrotron FU II submillimeter wave radiation source to plasma scattering measurement, Int. J. Infrared and Millimeter Waves 25 (2004) 1567-1579.

10) T. Tatsukawa, T. Shirai, T. Imaizumi, T. Idehara, I. Ogawa, and T. Kanemaki, Ruby ESR over a wade frequency range in the millimeter wave region, Int. J. Infrared and Millimeter Waves 19 (1998) 859-874.

11) T. Tatsukawa, A. Doi, M. Teranaka, H. Takashima, F. Goda, T. Idehara, T. Kanemaki, S. Nishizawa and T. Namba, Characteristics of a Teflon Rod Antenna for Millimeter- and Submillimeter- Wave Irradiation on Living Bodies, Jpn. J. Appl. Phys. 42 (2003) 7147-7150.

12) T. Idehara, S. Mitsudo and I. Ogawa, Development of high frequency, high stable gyrotrons as millimeter to submillimeter wave radiation sources, IEEE Trans. Plasma Sci. 32 (2004) 910-916.

13) T. Idehara, M. Pereyaslavets, N. Nishida, K. Yoshida, and I. Ogawa , Frequency modulation in a submillimeter-wave gyrotron, Phys., Rev. Lett. 81 (1998) 1973-1976.

14) T. Idehara, Y. Shimizu,S. Makino, K. Ichikawa, T. Tatsukawa, I. Ogawa, and G.F. Brand, High-frequency, amplitude modulation of a submillimeter wave, medium power gyrotron, Phys. Plasma 1 (1994) 461-463.

15) I. Ogawa, T. Idehara, M. Ui, S. Mitsudo and W. Feurster, Stabilzation and modulation of the output power of submillimeter wave gyrotron, Fusion Eng. and Design 53 (2001) 571-576.

16) T. Idehara, I. Ogawa and S. Mitsudo, Y. Iwata, S. atanabe, Y. Itakura, K. Ohashi, H. Kobayashi, T. Yokoyama, V. Zapevalov, M. Glyavin, A. Kuftin, O. Malygin and S. Sabchevski, Developmnt of a high harmonic gyrotron with an axis-encircling electron beam and a permanent magnet, Vacuum 77 (2005) 539-546. 
17) T. Idehara, N. Nishida, K. Yoshida, I. Ogawa, T. Tatsukawa, D. Wagner, G. Gantenbein, W. Kasparek, and M. Thumm, High frequency and high mode purity operations of Gyrotron FU IVA, Int. J. Infrared and Millimeter Waves 19 (1998) 919-930.

18) I. Ogawa, T. Idehara, T. Okada, S. Maeda, Y. Iwata, R. Pavlichenko, S. Mitsudo, D. Wagner and M. Thumm, High Quality Operation of a Submillimeter Wave Gyrotron for Plasma Diagnostics Application, J. Plasma and Fusion Research Series 5 (2003) 205-209. 